\title{
Heat Conduction and Geometry Topology Optimization of Support Structure in Laser-based Additive Manufacturing
}

\author{
Ehsan Malekipour ${ }^{1}$, Andres Tovar $^{2}$, Hazim El-Mounayri ${ }^{3}$ \\ Graduate Student ${ }^{1}$, Assistant Professor ${ }^{2}$, Associated Professor ${ }^{3}$ \\ Center for Additive Manufacturing Research at IUPUI (CAMRI) \\ Purdue School of Engineering and Technology, Indianapolis, IN, USA \\ emalekip@purdue.edu ${ }^{1}$,tovara@iupui.edu²,helmouna@iupui.edu ${ }^{3}$
}

\begin{abstract}
:
Laser-based metal additive manufacturing technologies such as Selective Laser Sintering (SLS) and Selective Laser Melting (SLM) allow the fabrication of complex parts by selectively sintering or melting metallic powders layer by layer. Although elaborate features can be produced by these technologies, heat accumulation in overhangs leads to heat stress and warping, affecting the dimensional and geometrical accuracy of the part. This work introduces an approach to mitigate heat stress by minimizing the temperature gradient between the heat-accumulated zone in overhangs and the layers beneath. This is achieved by generating complex support structures that maintain the mechanical stability of the overhang and increase the heat conduction between these areas. The architecture of the complex support structures is obtained by maximizing heat conduction as an objective function to optimize the topology of support structure. This work examines the effect of various geometries on the objective function in order to select a suitable one to consume less material with almost same conduction. Ongoing work is the development of an experimental testbed for verification.
\end{abstract}

Keywords: Additive Manufacturing, laser-based Additive Manufacturing, support structure, topology optimization, heat stress

Introduction

Laser-based additive manufacturing (AM) is a manufacturing process in which a high power laser sinters/melts metal powders together [1-3]. Residual stress is one of the most frequent defect affecting significantly the precision of the process. The predominated reason for residual stress is heat stress because of temperature gradient existed in different zones [4-6]. This residual stress eventually leads to distortion and it may fail the whole process, which is significantly costly and consumes excess time. Nowadays manufacturers predominantly designs support structures to control distortion inside the part by increasing the resistance of the structure [7,8]. This approach, as a trial and error method, significantly depends upon the designers' experiments [8]. Thus, first, there is no way to verify the efficiency of the designed support structure before fabrication of the part and second, there is no standard criteria/approach to follow for different geometries. As Figure 1 shows, fabrication of support structures significantly effects on temperature uniformity, especially in overhangs [9] by increasing of heat conductivity and as a result, decreasing of temperature gradient. This specification of support structures can be employed efficiently to decrease heat stress in the fabricated part and eventually, reduces or eliminates distortion. More uniform temperature distribution also leads to more uniform microstructure. By generating more support structures (the dots in Figure 1.a show the positions of support structures), more uniform temperature distribution we can achieved (Figure 1.b) which means less temperature gradient. In addition, the temperature average decreases because of better conductivity. [9]

This is the author's manuscript of the article published in final edited form as:

Malekipour, E., Tovar, A., \& El-Mounayri, H. (2018). Heat Conduction and Geometry Topology Optimization of Support Structure in LaserBased Additive Manufacturing. In Mechanics of Additive and Advanced Manufacturing, Volume 9 (pp. 17-27). Springer, Cham.

https://doi.org/10.1007/978-3-319-62834-9_4 


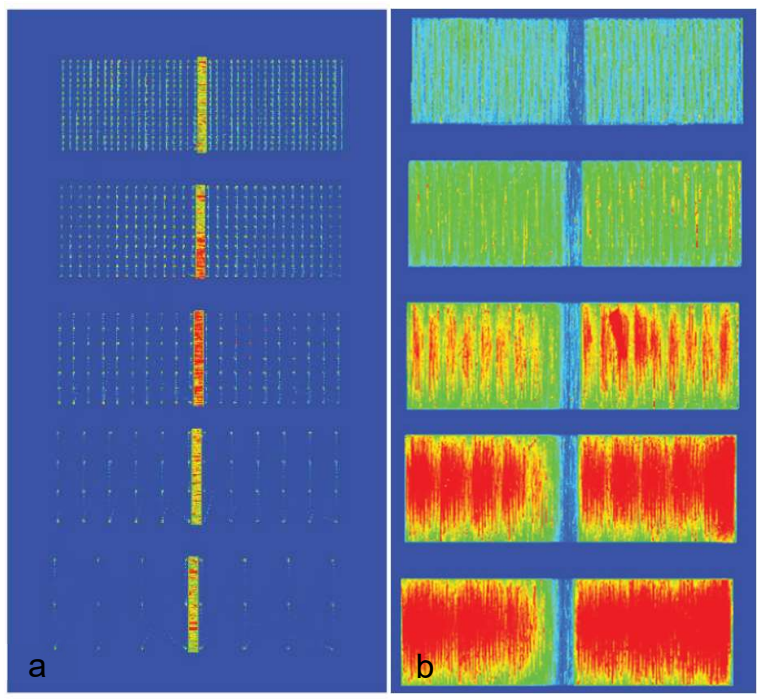

Figure 1: effect of support structure on temperature distribution and temperature gradient [9]

In this regard, heat conduction in topology optimization is employed to design architecture of complex support structures. The top3d 169 lines code [10] is employed and modified here. The methodology is explained in the following section.

\section{Methodology}

As it is explained in the introduction, there is a correlation between the number of fabricated support structures connecting overhang and its previous fabricated layer, and temperature distribution in a way that fabrication of enough number of support structures between an overhang and its previous layer, makes a temperature distribution uniform in the overhang layer [9]. If we assume we have enough number of support structures then, we have an overhang with a uniform temperature distribution. In this case, we can simplify the manufacturing model shown in figure 1.a with a simple support structure in Figure 2.b. In Figure 1.a an overhang exerted by the laser beam (the heat affected zone (HAZ)) in its corner for fabricating of the next layer was joined to its previous layer of fabricated part (the heat sink) by a simple rectangular column as the support structure (Figure 2.a). Due to temperature uniformity on the overhang, we assume the same temperature on top face of support structure; simultaneously, the its bottom face will be the heat sink because it connected to the bottom layer which also reached to a uniform temperature by passing time.
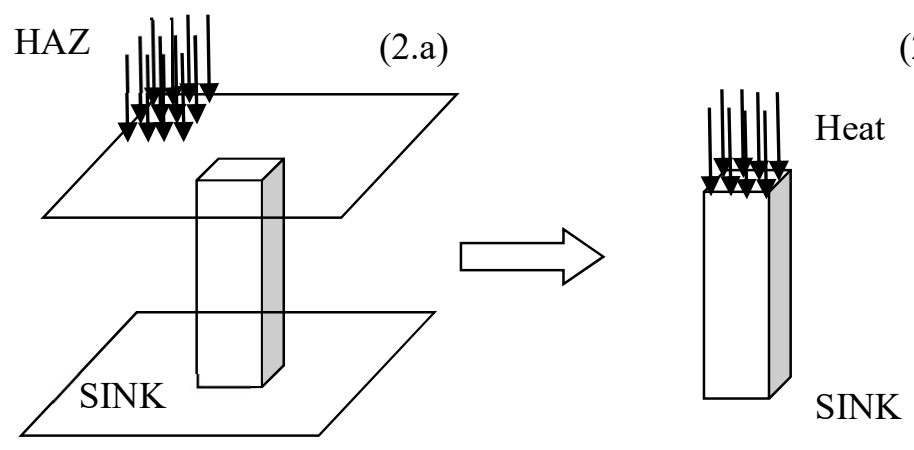

Figure 2: Model of the problem 2.a. Real model with overhang, support structure and the layer beneath; 2.b. Simplified model 
In this paper, we employs topology optimization to find the distribution of material in a prescribed area or volume referred to as the design domain by coding a binary programming problem introduced in reference [10]. In this regard, we employ topology optimization to maximize heat conduction in the designed support structures. Thus, manufacturers can fabricate the support structures with the same heat conductivity as the solid ones but with using fewer materials.

The equilibrium condition for heat transfer in finite element formulation is described by $\boldsymbol{K}\left(\boldsymbol{k}_{i}^{\mathbf{0}}\right) \boldsymbol{U}\left(\boldsymbol{k}_{i}^{\mathbf{0}}\right)=\boldsymbol{F}$ where $\boldsymbol{U}\left(\boldsymbol{k}_{i}^{\mathbf{0}}\right)$ shows the finite element global nodal temperature vector, F shows the global thermal load vector, and $\boldsymbol{K}\left(\boldsymbol{k}_{i}^{\mathbf{0}}\right)$ shows the global thermal conductivity matrix. The optimization problem for heat conduction is

Find

$$
\mathbf{K}_{\mathrm{i}}^{0}=\left[x_{1}, x_{2}, \ldots, x_{e}, \ldots, x_{n}\right]^{T}
$$

Minimize $\quad \mathrm{c}\left(\mathbf{K}_{\mathrm{i}}^{0}\right)=\mathbf{F}^{\mathrm{T}} \mathbf{U}\left(\mathbf{k}_{\mathbf{i}}^{0}\right)$

Subject to $\quad v(\widetilde{\boldsymbol{x}})=\widetilde{\boldsymbol{x}}^{T} \mathbf{v}-\bar{v} \leq 0$

$$
\mathbf{x} \in X, X=\left\{\mathbf{x} \in \mathrm{R}^{\mathrm{n}}: 0 \leq \mathbf{x} \leq 1\right\}
$$

Where $\mathbf{U}(\tilde{\mathbf{x}})=\mathbf{K}(\tilde{\mathbf{x}})^{-1} \mathbf{F}$, and $\mathbf{K}(\tilde{\mathbf{x}})$ is obtained by the assembly of elements in thermal conductivity problem which is expressed as:

$\boldsymbol{k}_{i}\left(\widetilde{x_{l}}\right)=\left[k_{\min }+{\widetilde{x_{l}}}^{p}\left(k_{0}-k_{\min }\right)\right] k_{i}^{0}$

Where $k_{\min }$ and $k_{0}$ represent the limits of the material's thermal conductivity coefficient and $k_{i}^{0}$ shows the element conductivity matrix. [10]

The primary top3d code solves minimum compliance for structural problems. By implementation of some modifications that are explained in following sentences, this code can also be used for heat conduction problem. The first modification is in heat conduction problem the number of DOF per node is one rather than three in minimum compliance in structure problems. The location of supports and applied forces also can be modified by considering the relationships between node number, node coordinates, node ID and node DOFs (Table 1).

Another modification is about creating various geometries by defining different active and passive elements. In this case, designers are able to calculate and compare the value of objective functions for different geometries and choose the support structure that consume fewer material but maximized objective function (heat conductivity) before the fabrication process. Objective function can be calculated by employing Optimality Criteria (OC) method. OC is a classical approach for solving a discretized structural optimization problem numerically. It is shown that this method is very efficient for solving the topology optimization problems. [11]

The power law penalizes intermediate density values implicitly and make the resulting structure more black-and-white. This penalization procedure is usually referred to as the Solid Isotropic Material with Penalization (SIMP) method [12]. It should be mentioned that we consider the penalization constant and equal to 1.5 for all of the generated model in the future to eliminate the effect of varied penalization on the final results. A basic filter density function is defined as:

$x_{i}^{\sim}=\frac{\sum_{j \in N_{i}} H_{i j} v_{j} x_{j}}{\sum_{j \in N_{i}} H_{i j} v_{j}}$

Where $N i$ is the neighborhood of an element $x_{i}$ with volume $v_{i}$, and $H_{i j}$ is a weight factor. The filtered density $x_{i}^{\sim}$ indicates a modified (physical) density field. The modified conductivity used for heat conduction problem is:

$$
\boldsymbol{k}_{i}\left(\widetilde{x_{l}}\right)=\left[k_{\min }+{\widetilde{x_{l}}}_{i}^{p}\left(k_{0}-k_{\min }\right)\right] k_{i}^{0} \quad x_{i}^{\sim} \in[0,1]
$$


Table 1: Illustration of relationships between node number, node coordinates, node ID and node DOFs [10]

\begin{tabular}{|c|c|c|c|c|c|}
\hline \multirow[t]{2}{*}{ Node Number } & \multirow[t]{2}{*}{ Node coordinates } & \multirow[t]{2}{*}{ Node ID } & \multicolumn{3}{|c|}{ Node Degree of Freedoms } \\
\hline & & & $\mathrm{x}$ & $\mathrm{y}$ & $\mathrm{z}$ \\
\hline$N_{1}$ & $\left(x_{1}, y_{1}, z_{1}\right)$ & $\operatorname{NID}_{1}^{\dagger}$ & $3 * \operatorname{NID}_{1}-2$ & $3 * \mathrm{NID}_{1}-1$ & $3 * \mathrm{NID}_{1}$ \\
\hline $\mathrm{N}_{2}$ & $\left(x_{1}+1, y_{1}, z_{1}\right)$ & $\mathrm{NID}_{2}=\mathrm{NID}_{1}+($ nely +1$)$ & $3 * \mathrm{NID}_{2}-2$ & $3 * \mathrm{NID}_{2}-1$ & $3 * \mathrm{NID}_{2}$ \\
\hline$N_{3}$ & $\left(x_{1}+1, y_{1}+1, z_{1}\right)$ & $\mathrm{NID}_{3}=\mathrm{NID}_{1}+$ nely & $3 * \mathrm{NID}_{3}-2$ & $3 * \mathrm{NID}_{3}-1$ & $3 * \mathrm{NID}_{3}$ \\
\hline$N_{4}$ & $\left(x_{1}, y_{1}+1, z_{1}\right)$ & $\mathrm{NID}_{4}=\mathrm{NID}_{1}-1$ & $3 * \mathrm{NID}_{4}-2$ & $3 * \mathrm{NID}_{4}-1$ & $3 * \mathrm{NID}_{4}$ \\
\hline$N_{5}$ & $\left(x_{1}, y_{1}, z_{1}+1\right)$ & $\mathrm{NID}_{5}=\mathrm{NID}_{1}+\mathrm{NID}_{2}^{\ddagger}$ & $3 * \mathrm{NID}_{5}-2$ & $3 * \mathrm{NID}_{5}-1$ & $3 * \mathrm{NID}_{5}$ \\
\hline$N_{6}$ & $\left(x_{1}+1, y_{1}, z_{1}+1\right)$ & $\mathrm{NID}_{6}=\mathrm{NID}_{2}+\mathrm{NID}_{z}$ & $3 * \mathrm{NID}_{6}-2$ & $3 * \mathrm{NID}_{6}-1$ & $3 * \mathrm{NID}_{6}$ \\
\hline$N_{7}$ & $\left(x_{1}+1, y_{1}+1, z_{1}+1\right)$ & $\mathrm{NID}_{7}=\mathrm{NID}_{3}+\mathrm{NID}_{z}$ & $3 * \mathrm{NID}_{7}-2$ & $3 * \mathrm{NID}_{7}-1$ & $3 * \mathrm{NID}_{7}$ \\
\hline$N_{8}$ & $\left(x_{1}, y_{1}+1, z_{1}+1\right)$ & $\mathrm{NID}_{8}=\mathrm{NID}_{4}+\mathrm{NID}_{z}$ & $3 *$ NID $_{8}-2$ & $3 * \mathrm{NID}_{8}-1$ & $3 * \mathrm{NID}_{8}$ \\
\hline
\end{tabular}

${ }^{+} \mathrm{NID}_{1}=z_{1} *($ nel $\mathrm{x}+1) *($ nel $\mathrm{y}+1)+x_{1} *($ nel $\mathrm{y}+1)+\left(\right.$ nely $\left.+1-y_{1}\right)$

${ }^{\ddagger} \mathrm{NID}_{z}=(\mathrm{nelx}+1) *(\mathrm{nel} \mathrm{y}+1)$

It should be noted that the traditional direct solver does not work expeditiously for the finite element large-scale problems. Nevertheless, iterative solver can solve large-scale problems efficiently [13, 14]. In this regard, line 72 of top3d code is replaced by a built-in MATLAB function pcg, called preconditioned conjugate gradients method.

\section{Result and Discussion}

As it was already explained the main objective is to optimize the topology of a simple rectangular column as a support structure between an overhang and its previous layer. As Figure 3.a shows, the primary model in top3d code for heat conduction includes a rectangular cube, which has a sink on the middle of top surface, and all other nodes are given a thermal load.
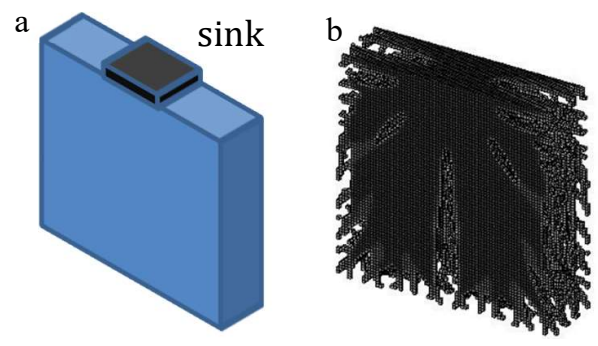

Figure 3: a. The primary model for support structure; b. The resulting topology optimized shape

Figure 3.b shows the resulting shape for this case. In the next step, we change the geometry with using different pattern of active and passive elements (see Appendix, section: DEFINING PASSIVE ELEMENTS Status, line 70 to 131) to find the geometry generating minimum objective function and as a result, maximum heat conductivity. Finally, the boundary conditions (heat flux and sink) are applied on the obtained geometry to model the desired laser-based AM process. It is noteworthy that for eliminating the effect of other parameters on the final result, we keep the volume fraction equals to 0.4 , penalization equals to 3 , minimum radius filter ( $r$ min) equals to 1.5 , number of iteration equals to 60 and dimensions equals to $80 \times 80 \times 20$ fixed for all tests. 
Table 2: Generating various geometries by using different patterns of active and passive elements

\begin{tabular}{|c|c|c|c|}
\hline No. & Geometrical Type & Value of Objective Function & Resulting Shape \\
\hline 1 & Primary model & Obj.: 97580.9442 & \\
\hline 2 & Primary model set limits on two sides & Obj.:189254.3599 & \\
\hline 3 & $\begin{array}{l}\text { Primary model with a big hole at the } \\
\text { middle }\end{array}$ & Obj.:332743.6824 & \\
\hline 4 & $\begin{array}{l}\text { Primary model with a small hole at } \\
\text { the middle }\end{array}$ & Obj.:109929.4235 & \\
\hline 5 & $\begin{array}{l}\text { Primary model with a small hole at } \\
\text { the middle top }\end{array}$ & Obj.:134462.7200 & \\
\hline 6 & $\begin{array}{l}\text { Primary model with two small holes } \\
\text { at the middle top and middle bottom }\end{array}$ & Obj.:148970.7845 & \\
\hline
\end{tabular}

Table 2 shows that the value of objective function is minimum for the geometry without any passive elements (any holes). This result is predictable because solid support structure conducts heat better than any other types of geometry. But the 
best option is the fourth one since the criteria for choosing the most optimized support structure is the one with minimum objective function (maximum conductivity) while consume less material. As Table 2 shows the percentage difference between the value of objective functions of number one and four is just around $11 \%$ while it uses less material. Thus, we choose the geometry type number four as the best one for support structure; The objective function variation for different types of geometry is revealed in the following diagram.

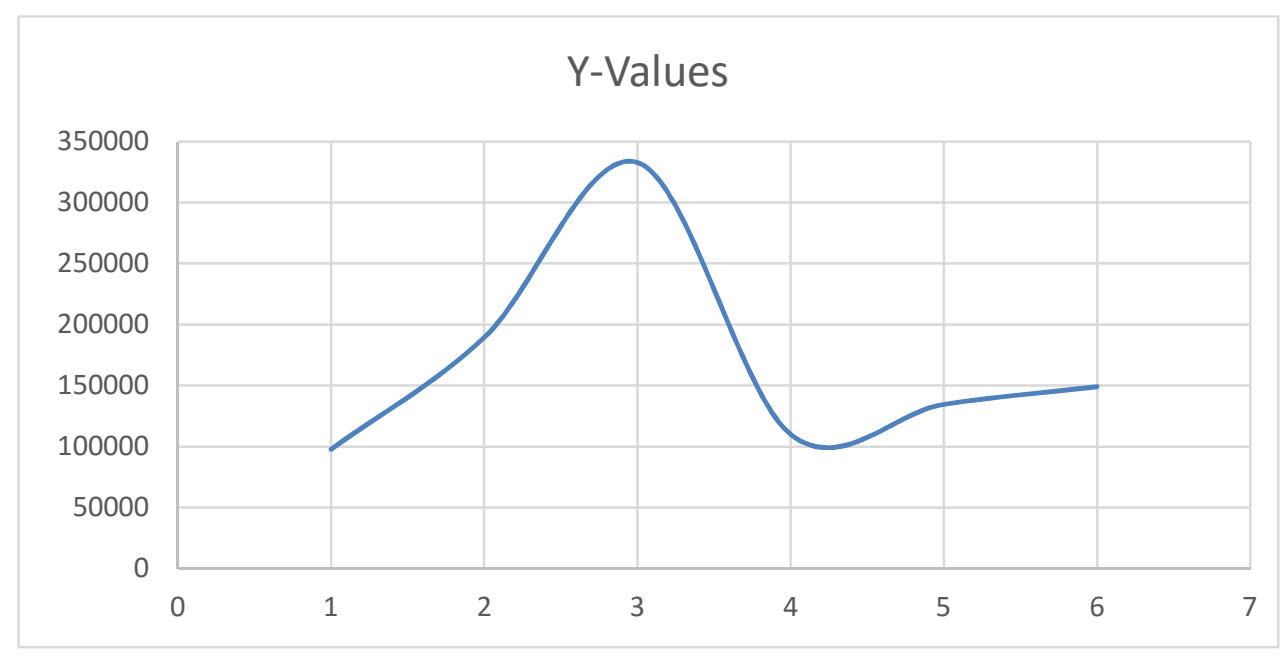

Figure 4: The value of objective function for different types of geometry revealed in table 2

As the final step, the desired boundary conditions (exerted heat and sink zone) are applied on the chosen geometry (see Appendix, section: Different load conditions, line 24 to 29) and the resulting shape of support structure are derived. This shape is the optimized support structure that should be used by vendors in the specified manufacturing conditions.

Table 3: Optimized support structure for different boundary conditions

\begin{tabular}{lccc}
\hline No. & Boundary Conditions & Value of Objective Function & Optimized Support Structure \\
$\mathbf{1} \quad \begin{array}{c}\text { sink is placed at the bottom } \\
\text { face }\end{array}$ & Obj.:109614.2276 \\
$\mathbf{2} \quad \begin{array}{c}\text { Heat flux is exerted on half } \\
\text { of the top face and sink is } \\
\text { placed at the bottom face }\end{array}$ & Obj.:274029098.3609
\end{tabular}


Heat flux is exerted on the

\section{Summery and conclusions}

This paper shows how manufacturers can optimize support structures topologically between overhangs and the layer beneath, in order to maximize heat conduction subjected to structural stiffness constraint in laser-based AM.

In this regard, top3d 169 lines MATLAB code is modified and employed. Firstly, various geometries are generated by defining different patterns for active and passive elements and then, the best geometry is identified based on maximum heat conduction while using less material. The results show that the value of objective function for the geometry with a small hole in the middle of structure is minimum showing maximum heat conduction. Finally, different boundary conditions with respect to different fabrication circumstances are applied and the final topological optimized shape for support structures are derived. It should be noticed that in this paper we just examined four different types of geometry (Table 2) and also, utilize mono-physical objective function. As the next step, we are going to examine more types of geometries and also, utilize multi-physics objective function in which heat conduction is maximized subjected to structural stiffness constraint and finally, development of an experimental testbed for verification.

\section{References}

[1] D. Gu, W. Meiners, K. Wissenbach, and R. Poprawe, "Laser additive manufacturing of metallic components: materials, processes and mechanisms," International materials reviews, vol. 57, pp. 133164, 2012.

[2] V. Bhavar, P. Kattire, V. Patil, S. Khot, K. Gujar, and R. Singh, "A Review on Powder Bed Fusion Technology of Metal Additive Manufacturing," in International Conference \& Exhibition on Additive Manufacturing Technologies Bangalore, India, 2015.

[3] W. E. Frazier, "Metal additive manufacturing: a review," Journal of Materials Engineering and Performance, vol. 23, pp. 1917-1928, 2014.

[4] M. F. Zaeh and G. Branner, "Investigations on residual stresses and deformations in selective laser melting," Production Engineering, vol. 4, pp. 35-45, 2010.

[5] I. A. Roberts, "Investigation of residual stresses in the laser melting of metal powders in additive layer manufacturing," 2012.

[6] B. Cheng and K. Chou, "Thermal Stresses Associated with Part Overhang Geometry in Electron Beam Additive Manufacturing: Process Parameter Effects," in Proc. Annu. Int. Solid Freeform Fabr. Symp, 2014, p. 1076. 
[7] T. Krol, M. Zäh, and C. Seidel, "Optimization of supports in metal-based additive manufacturing by means of finite element models," Bourell DL, 2012.

[8] F. Calignano, "Design optimization of supports for overhanging structures in aluminum and titanium alloys by selective laser melting," Materials \& Design, vol. 64, pp. 203-213, 2014.

[9] T. Craeghs, S. Clijsters, J.-P. Kruth, F. Bechmann, and M.-C. Ebert, "Detection of process failures in layerwise laser melting with optical process monitoring," Physics Procedia, vol. 39, pp. 753-759, 2012.

[10] K. Liu and A. Tovar, "An efficient 3D topology optimization code written in Matlab," Structural and Multidisciplinary Optimization, vol. 50, pp. 1175-1196, 2014.

[11] P. W. Christensen and A. Klarbring, An introduction to structural optimization vol. 153: Springer Science \& Business Media, 2008.

[12] M. Zhou and G. Rozvany, "The COC algorithm, Part II: Topological, geometrical and generalized shape optimization," Computer Methods in Applied Mechanics and Engineering, vol. 89, pp. 309-336, 1991.

[13] M. R. Hestenes and E. Stiefel, Methods of conjugate gradients for solving linear systems vol. 49: NBS, 1952.

[14] C. Augarde, A. Ramage, and J. Staudacher, "An element-based displacement preconditioner for linear elasticity problems," Computers \& structures, vol. 84, pp. 2306-2315, 2006. 


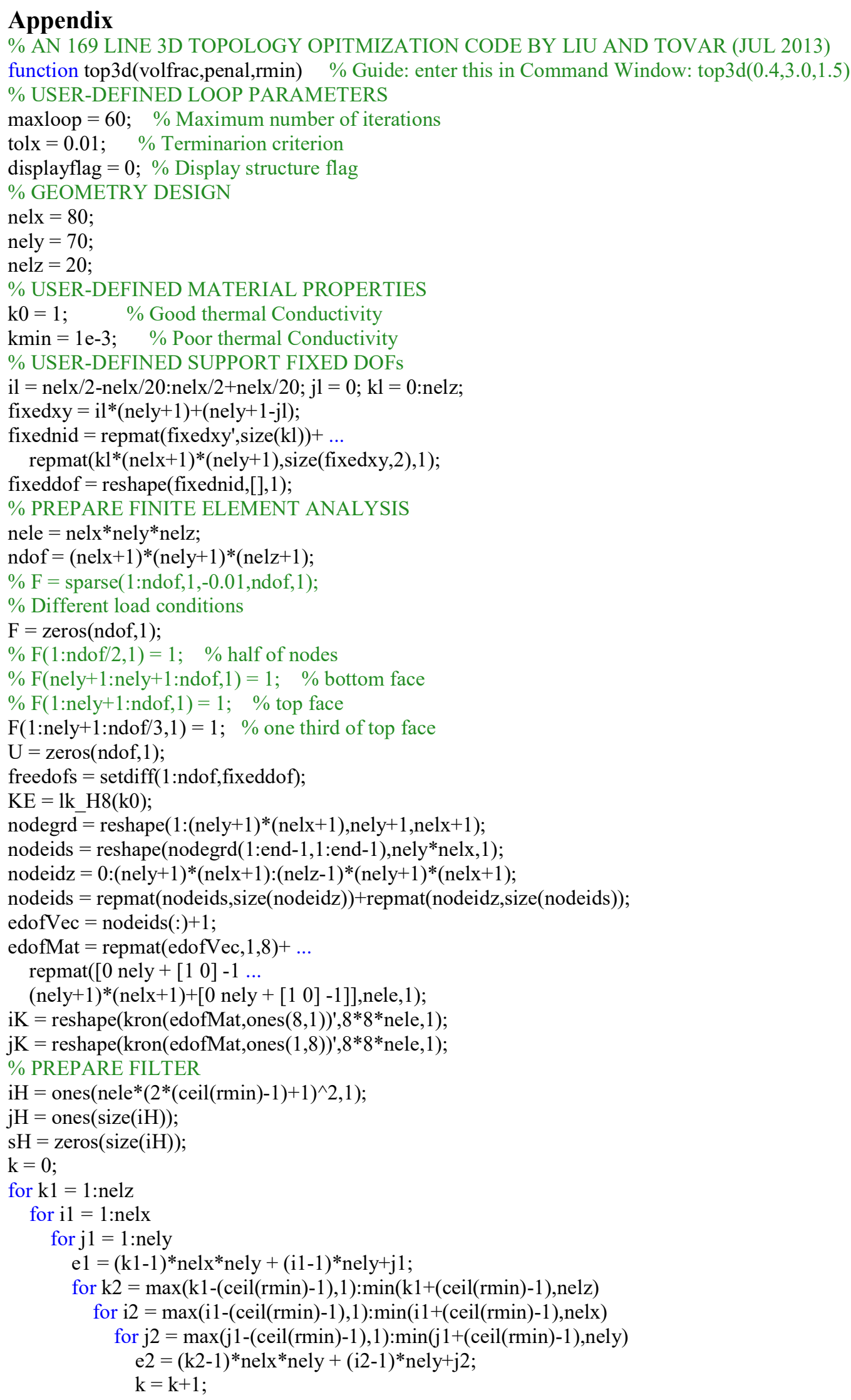




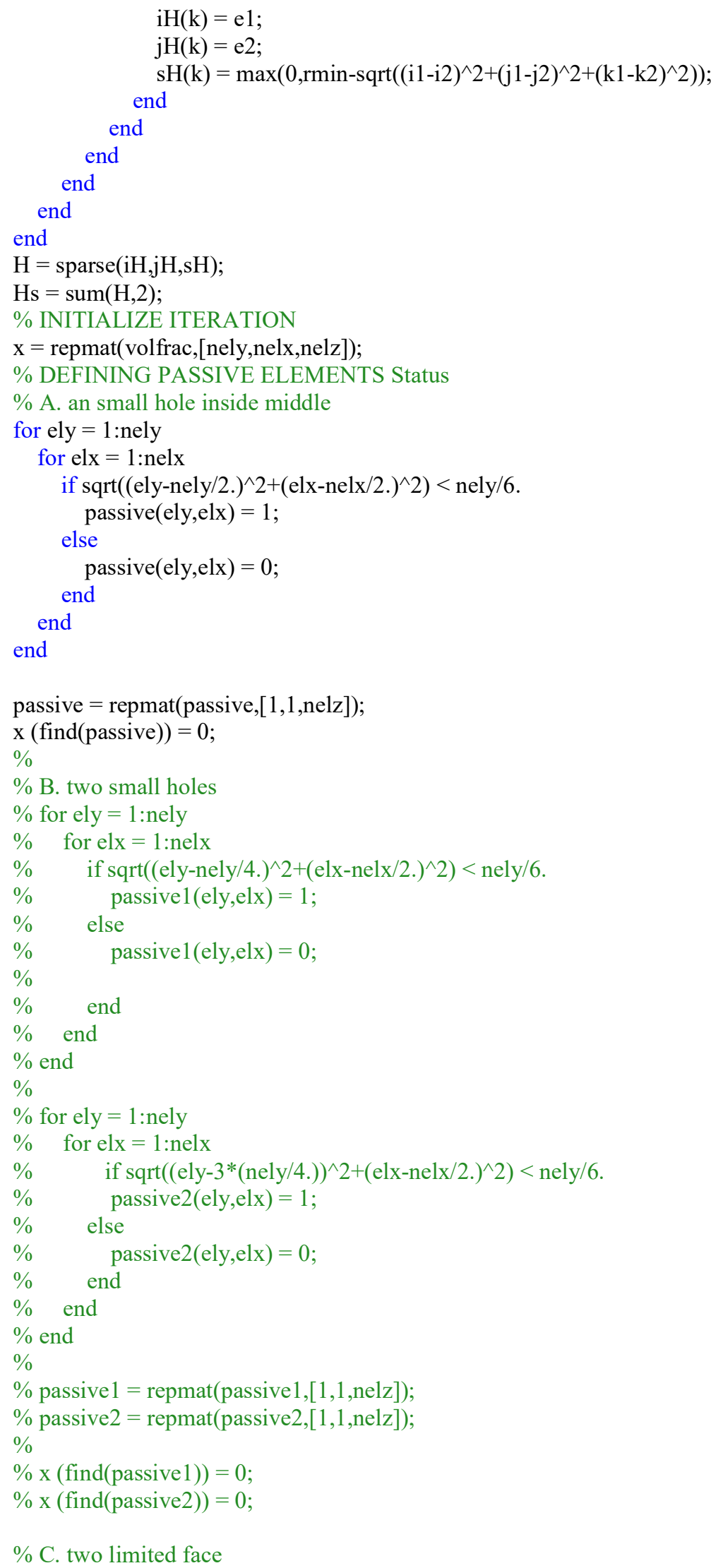




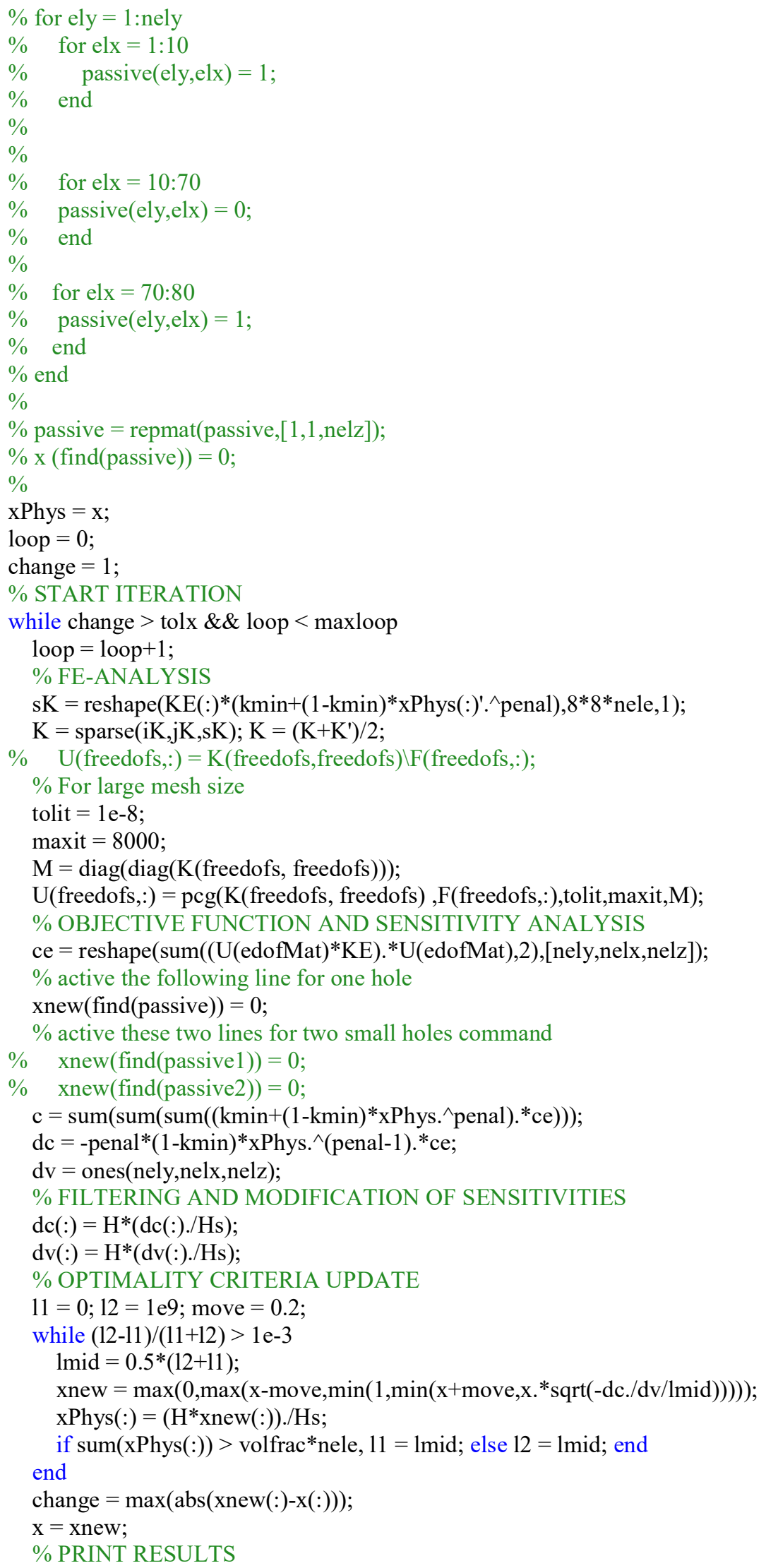


